\section{HACIA EL DISEÑO Y GESTIÓN DE BARRIOS SUSTENTABLES EN CHILE}

Juan Blanco Moya ${ }^{1}$

\section{Resumen}

Chile es un país en crecimiento, donde la provisión de vivienda ha impulsado un fuerte desarrollo en el mercado inmobiliario. En el año 2012, 3.500 unidades de vivienda se vendieron en el país. La mayoría de ellas asociada a la tipología de barrio. Este patrón de crecimiento se encuentra muchas veces vinculado a la expansión del suelo urbano, con un fuerte impacto en su entorno físico-natural y social, así como un incremento en el gasto de energía y materiales. Diversas estrategias se han efectuado para mitigar los efectos de este desarrollo, basadas en la idea de aplicar criterios de sustentabilidad a proyectos urbanos, particularmente a

\section{TOWARDS THE DESIGN AND MANAGEMENT OF SUSTAINABLE NEIGHBORHOODS IN CHILE}

Juan Blanco Moya ${ }^{1}$

\section{Abstract}

Chile is an emerging nation where the provision of housing has prompted the development of the real estate market. During 2012, 3,500 housing units were sold in the country. Most of these dwellings are associated with the neighborhood typology. Such a growth pattern is often related to the expansion of the city, has a strong impact on its physical-natural and social environments and implies an increase in the consumption of materials and energy. Different urban sustainability strategies —which are especially focused on new neighborhoods- have been 
partir de nuevos barrios. A pesar de ello, en el ámbito nacional no existe consenso respecto a cómo lograr barrios sustentables, y tampoco se cuenta con una herramienta de evaluación establecida con la cual medir el mérito de tales proyectos en términos de su contribución al desarrollo sustentable. El presente artículo se propone entender hasta qué punto estándares internacionales son factibles de ser implementados en proyectos nacionales de barrios, a partir de una revisión de los referentes internacionales más divulgados, para luego ahondar en sus ventajas y dificultades de ser transferidos al mercado inmobiliario.

\section{PALABRAS CLAVE: BARRIOS SUSTENTABLES; ESTÁNDARES DE SUSTENTABILIDAD; POLÍTICA URBANA}

1 Chile. Investigador asociado Universidad Autónoma de Chile. Correo electrónico: jpblancomoya@gmail.com. developed in order to mitigate the effects of this development. However, there is no national consensus on how to produce sustainable neighborhoods nor is there an assessment tool which might measure the contribution of these projects to sustainable development. Through the review of international sources this paper seeks to understand the extent to which international standards could be implemented in local neighborhood projects. Then, the advantages and difficulties related to the transferring of these standards to the real estate market are further explored.

KEYWORDS: SUSTAINABLE NEIGHBORHOODS; SUSTAINABILITY STANDARDS; URBAN POLICY.

Received: 16-02-2014

Accepted: 09-09-2015

1 Chile. Research associate, Autonomous University of Chile. Email: jpblancomoya@gmail.com.

OPINION: Towards the design and management of SuStainable neighborhoods in Chille 


\section{Barrios y comunidades sustentables}

Si bien el concepto de eco-eficiencia ha sido exitosamente aplicado a viviendas para ahorrar energía, mediante el aislamiento en paredes y ventanas reduciendo así la carga sobre los sistemas de aire acondicionado del edificio para su refrigeración y por ende disminuyendo la cantidad de energía utilizada proveniente de combustibles fósiles ${ }^{2}$, tal aproximación es insuficiente para el diseño del conjunto de viviendas y sus espacios comunes. En ese sentido, tanto la métrica como la gestión y diseño de una unidad eco-eficiente no siempre tienen correlación directa al momento de repetir dicha unidad para construir un conjunto, debido a que muchos atributos que pueden ser una ventaja a nivel individual, se vuelven redundantes o poco eficientes, como el uso de Energías Renovables No Convencionales (ERNC), espacio de estacionamiento, redes y orientación, entre muchos otros.

Actualmente se plantea la necesidad de repensar los sistemas urbanos desde posiciones próximas a la ecología académica. A su vez, la ecología urbana es una visión integradora del conjunto de disciplinas que intervienen sobre los sistemas urbanos ${ }^{3}$. La adaptación de conceptos provenientes del campo

2 Braungart y Mcdonough, 2005, p. 70.

3 Rueda, 1995, p. 60-68. de la física, como materia (el consumo y producción de materia por los sistemas urbanos), energía (el balance energético de la ciudad) einformación (u organización), al caso de las ciudades ha permitido un inicial conocimiento y diagnóstico del grado de eficiencia ambiental de las mismas en relación a otros ecosistemas en el marco del desarrollo sustentable. En ese contexto, el presente artículo de opinión parte de la premisa de contar con un instrumento que permita evaluar el aporte al desarrollo sustentable de iniciativas de barrios, tomando en cuenta que la práctica urbanística nacional no dispone de un instrumento para ello y, hasta el momento, tales mediciones se han realizado a través de estándares internacionales disponibles.

A modo de ejemplo, LEED ND es un sistema diseñado para certificar proyectos ejemplares que se comportan bien en términos de crecimiento inteligente, urbanismo y edificación verde. Además de crear una etiqueta, sirve de guía, tanto para la toma de decisiones como para el diseño y desarrollo, otorgando un incentivo a la correcta ubicación, diseño y construcción de nuevos proyectos tanto residenciales como comerciales y de uso mixto. A su vez, BREEAM Communities tiene una aproximación similar en cuanto es un sistema basado en créditos, pero incorpora una serie de aspectos que lo hacen relevante al momento de considerar su 
implementación a nivel local. En primer lugar, la manera como se establecen los límites de barrios o comunidades urbanas es diferente. BREEAM permite considerar tamaños desde 10 unidades (proyectos pequeños) a 6.000 unidades (proyectos grandes) como comunidades urbanas; sin embargo, también considera proyectos de más de 6.000 unidades, luego de la aprobación del British Research Establishment (BRE), entidad que administra esta certificación. LEED sugiere considerar barrios con una extensión no superior a $1.3 \mathrm{~km} 2$, con la posibilidad de dividir el proyecto si la superficie excede dicho valor. Por ejemplo, proyectos piloto que han sido evaluados con LEED ND tienen un promedio de tamaño de $1.2 \mathrm{~km} 2$. En específico, el tamaño menor fue de $687 \mathrm{~m} 2$, mientras que el más grande fue de $51.8 \mathrm{~km} 2^{4}$.

Respecto a la ponderación en la categoría de "localización", ésta está probablemente influenciada por los distintos marcos de aplicación de los sistemas revisados. LEED ND tiene como objetivo ser aplicado a nuevos barrios, en cambio BREEAM Communities está fundamentalmente enfocado en intervención de rehabilitación. Estos campos de operación diferentes han probablemente desalentado

4 U.S. Green Building Council, 2014, p. 12. la asignación de mayor ponderación a la localización en BREEAM Communitites, debido a que esta categoría de evaluación está generalmente satisfecha en proyectos de regeneración o rehabilitación de barrios, localizados en áreas centrales o peri-centrales.

Por otro lado, Green Star Communities (sistema disponible en el mercado en su primera versión piloto desde fines 2012) posee un marco de acción nacional, identificando objetivos y aspectos a evaluar para medir la sustentabilidad de comunidades. Este sistema pretende enfatizar en la habitabilidad, intentando reconocer a aquellos proyectos que buscan el desarrollo de comunidades socioeconómicamente diversas, asequibles, saludables y seguras, diseñadas para albergar variados usos y actividades, que fomentan la inclusión de personas de todas las edades y culturas y que facilitan la cohesión, participación ciudadana y adaptabilidad de la comunidad. Además busca impulsar la innovación, promoviendo la eficiencia y efectividad en el uso de recursos, atraer inversión y estimular la producción y consumo de productos locales, así como promover la coordinación y el involucramiento de los stakeholders. 
TABLA 1. LEED ND, BREEAM COMMUNITIES Y GREEN STAR COMMUNITIES. CRITERIOS Y PUNTUACIÓN.

\begin{tabular}{|c|c|c|c|}
\hline & $\begin{array}{l}\text { LEED ND (2009) } \\
\text { (Leadership in Environmental and } \\
\text { Energy Design) }\end{array}$ & $\begin{array}{l}\text { BREEAM Communities } \\
\text { (2012) } \\
\text { (BRE Environmental } \\
\text { Assessment Method) }\end{array}$ & $\begin{array}{l}\text { Green Star Communities } \\
{ }^{*} \text { versión piloto } 0.2 \\
\text { (2012-2015) } \\
\text { (Green Building Council of } \\
\text { Australia) }\end{array}$ \\
\hline COMPONENTES & $\begin{array}{l}\text { - Localización inteligente y redes }(25 \%) \\
\text { - Patrones de barrio y diseño }(40 \%) \\
\text { - Infraestructura verde y edificios }(26 \%) \\
\text { - Innovación y proceso de diseño }(5 \%) \\
\text { - Prioridad regional }(4 \%)\end{array}$ & $\begin{array}{l}\text { - Gobernanza ( } 9 \%) \\
\text { - Bienestar social y económico } \\
(42 \%) \\
\text { - Recursos y energía }(22 \%) \\
\text { - Uso de suelo y ecología (13\%) } \\
\text { - Transporte y movilidad (14\%) }\end{array}$ & $\begin{array}{l}\text { - Gobernanza (19\%) } \\
\text { - Diseño }(9 \%) \\
\text { - Habitabilidad (16\%) } \\
\text { - Prosperidad económica (18\%) } \\
\text { - Medio ambiente }(28 \%) \\
\text { - Innovación }(9 \%)\end{array}$ \\
\hline CERTIFICACIÓN & 110 puntos ( $80+$ platino) & 100 puntos $(70 \%+=$ excelencia $)$ & $\begin{array}{l}110 \text { puntos }(75 \%+=\text { líder } \\
\text { mundial) }\end{array}$ \\
\hline
\end{tabular}

Fuente: autor en base a manuales técnicos de LEED, BREEAM y Green Star.

Junto con los estándares revisados, también cabe destacar la experiencia One Planet Living $(\mathrm{OPL})^{5}$. Esta iniciativa está liderada por el consorcio Biorregional y ha sido desarrollada desde la experiencia pionera del barrio de BedZED Eco-Villa, localizado al sur de Londres. A partir de la experiencia recogida en este proyecto, busca promover una forma más asequible y directa para difundir estilos de vida más sustentables, más allá de la generación de viviendas sustentables, es decir, a través de la incorporación de una infraestructura más am-

5 One Planet Living, s.f. plia, incluyendo la generación de nuevos servicios y productos. Desde su creación en 2003, OPL ha divulgado 10 principios para planificar, entregar y comunicar estrategias de desarrollo sustentable para barrios y comunidades.

Así, de la revisión presentada en esta sección, y tomando como referencia a Kellett ${ }^{6}$ y $\mathrm{Haapio}^{7}$, se pueden establecer los siguientes criterios que deberían abarcar cualquier sistema de evaluación para barrios, independiente de su contexto geográfico, político y social:

$\begin{array}{ll}6 & \text { Kellett, } 2009 . \\ 7 & \text { Haapio, } 2012 .\end{array}$ 
- Terreno sustentable, donde se incorporan temáticas como planificación sustentable, diseño y edificaciones y microclimas.

- Localización, uso de suelo previo y reducción de expansión urbana (sprawl).

- Transporte, incorporando al peatón, a los ciclistas y al transporte público.

- Energía y recursos, uso y selección de materiales, gestión de residuos y eficiencia en producción de energía.

- Ecología, referida fundamentalmente a preservar la biodiversidad.

- Economía y negocios, empleo y nuevas oportunidades.

- Bienestar, entendido como calidad de vida.

\section{Problemas de sistemas de certificación a escala barrial}

En primer lugar, los sistemas de certificación revisados muestran fuertes vínculos con sus contextos nacionales originales (Estados Unidos, Reino Unido y Australia) y no son lo suficientemente flexibles como para ser aplicados en otros ámbitos ${ }^{8}$. Es

8 Haapio, 2012, p. 166-169. por ello que estos sistemas no son necesariamente transferibles a otro contexto9. A pesar de ello, solo BREEAM Communities tiene procesos dedicados a responder temáticas locales que pudiesen alterar el contenido técnico de la herramienta (como valores asignados a criterios, o dificultad para trabajar con ciertos indicadores) y, así, alterar los límites dentro de los cuales un barrio puede demostrar progreso si deseara alcanzar dicha certificación. BREEAM Communities emplea un proceso de "ajuste internacional" (o International Bespoke) para evaluar la aplicabilidad de la certificación a proyectos internacionales (fuera del Reino Unido) y negociar cambios basado en legislación local, cultura, clima, etc. LEED ND enfrenta esta limitación de una manera distinta, por ejemplo al ofrecer pequeñas modificaciones de diferentes áreas para premiar un pequeño número de créditos por incorporar temáticas locales. Asimismo, la incorporación de actores relevantes es importante para entender las variaciones locales en una escala interna y regional. LEED ND no tiene como requerimiento el sostener reuniones con la comunidad local y autoridades públicas, o generar instancias de interacción con actores relevantes; BREEAM Communities sí tiene este requerimiento, a través del requisito por la ejecución de una sesión de pre-planificación con una variedad de actores locales y además incluye un tópico dedicado a "vínculo con la comunidad",

$9 \quad$ Garde, 2009, p. 435. 
trabajo que debe ser transferido a estrategias de diseño que impacten el layout final del proyecto.

En segundo lugar, si bien la evidencia internacional sugiere que el uso de estas certificaciones ayuda a generar desarrollos más sustentables a partir de la mejora desde una condición base ${ }^{10}$, la mayoría de estas investigaciones se focalizan en el desempeño de criterios individuales en la etapa de entrega de los proyectos, particularmente en términos de reducción de emisiones de carbono y uso de energía. Asimismo, existe poca literatura disponible respecto del desempeño de estos sistemas en la escala de barrio. También es importante destacar que algunos autores sugieren que los beneficios más significativos de edificios certificados tienden a ser "más sicológicos que empíricos"11, lo que sugiere que mayores esfuerzos de investigación deben ser realizados para ir más allá de la simple comparación de métricas. Para el caso específico de desarrollos urbanos certificados, la literatura plantea que no necesariamente se desempeñan cuantitativamente mejor que desarrollos convencionales, debido a una variedad de factores: los procesos o elementos de la etapa de diseño pudiesen no funcionar según

10 Lam, Chan, Poon, Chauc y Chun, 2010, p. 58-59.

11 Zuo y Zhao, 2014, p. 279. lo planificado ${ }^{12}$, los usuarios pudiesen interactuar con el barrio construido de forma inesperada, las especificaciones de los estándares pudiesen no ser mayores a las buenas prácticas ya instaladas en la oferta ${ }^{13}$, y desarrollados y diseñadores pudiesen perseguir criterios diferentes contra los cuales se implementa la medición ${ }^{14}$.

\section{Iniciativas de evaluación de barrios en el ámbito local}

Según Márquez ${ }^{15}$, en los últimos años ha surgido en Chile una revalorización del concepto de barrio, lo cual se plasma en varias políticas sociales, como por ejemplo el programa impulsado por el segundo gobierno de Michelle Bachelet de intervención en doscientos barrios vulnerables, "Quiero Mi Barrio" (en su segunda versión) $)^{16}$. Para Márquez, "en tiempos de debilitamiento del tejido social urbano, el concepto de barrio resurge desde la oferta inmobiliaria orientada a sectores medios altos"17. El barrio, en consecuencia, se ha posicionado como un concepto recurrente dentro de nuestro imaginario urbano, asociándolo con una recuperación

\footnotetext{
Abdalla, Maas, Huyghe y Oostra, 2011, p. 443-446.

Schweber, 2013, p. 12.

Sharifi y Murayama, 2013, p. 77.

Márquez, 2006, p. 38.

El programa Quiero Mi Barrio original se implementó entre 20062009 e incluyó intervenciones en 200 barrios vulnerables.

17 Márquez, 2006, p. 38.
} 
de estos espacios más tradicionales y de una vida más amable. En ese sentido, y a pesar de que en la práctica urbanística nacional se pueden encontrar diversas definiciones respecto al barrio, en Chile no existe una división administrativa de éstos. Sin embargo, se cuenta con algunos precedentes relevantes desde el contexto de las políticas públicas, particularmente desde experiencias de gestión medioambiental al interior de barrios, como los denominados Ecobarrios ${ }^{18}$. A su vez, el Programa Quiero Mi Barrio del Ministerio de Vivienda y Urbanismo del Gobierno de Chile se ha focalizado en la rehabilitación de barrios suburbanos de bajos recursos, la mayoría de ellos construidos en las décadas de los 80 s y 90 s. Es un programa basado en la comunidad cuyo objetivo es localizar recursos para el mejoramiento del espacio público y proyectos de infraestructura. Está basado en un set de criterios desarrollados para responder a las necesidades de la comunidad y del medio ambiente, en un esfuerzo por traducir principios del desarrollo sustentable a sectores vulnerables. Asimismo, ha desarrollado una herramienta para evaluar la línea de base de la comunidad y su barrio a partir de cinco criterios, con algunos de ellos relacionados a los estándares LEED ND, BREEAM Communities y Green Star Communities antes revisados, con una clara orientación hacia la participación ciudadana y el bienestar (tabla 2).

TABLA 2. COMPARACIÓN ENTRE SELECCIÓN DE CATEGORÍAS LEED, BREEAM Y GREEN STAR Y PROGRAMA QUIERO MI BARRIO.

\begin{tabular}{|c|c|c|}
\hline & $\begin{array}{l}\text { SELECCIÓN DE CATEGORÍAS } \\
\text { (Selección de LEED ND, BREEAM Communities y Green Star } \\
\text { Communities) }\end{array}$ & $\begin{array}{l}\text { Programa Quiero Mi Barrio II (2014) } \\
\text { (Ministerio de Vivienda y Urbanismo, MINVU) }\end{array}$ \\
\hline COMPONENTES & $\begin{array}{l}\text { - Terreno sustentable } \\
\text { - Localización } \\
\text { - Transporte } \\
\text { - Energía y recursos } \\
\text { - Ecología } \\
\text { - Economía y negocios } \\
\text { - Bienestar }\end{array}$ & $\begin{array}{l}\text { - Conectividad del barrio e integración territorial } \\
(20 \%) \\
\text { - Cohesión, recreación y agrupación de la comu- } \\
\text { nidad }(37 \%) \\
\text { - Prevención de amenazas y sustentabilidad } \\
\text { medioambiental }(14 \%) \\
\text { - Belleza, patrimonio y refuerzo de la identidad } \\
\text { local }(12 \%) \\
\text { - Seguridad urbana integral }(17 \%)\end{array}$ \\
\hline
\end{tabular}

Fuente: autor en base a manuales técnicos de LEED, BREEAM, Green Star Communities y MINVU.

18 Díaz, 2011, p. 114. 


\section{Conclusiones}

Respecto a las ventajas y dificultades de implementar los estándares revisados en Chile, el momento económico, político y social plantea diversos escenarios. En términos económicos, las proyecciones del crecimiento de la industria de la construcción son moderadas (estimaciones de ventas nacionales a 70.800 unidades en 2014, 3,6\% menos que en 2013) ${ }^{19}$, aunque el crecimiento a partir de barrios y comunidades sigue siendo un patrón de consumo requerido por el mercado. Sin embargo, no existe interés mayor desde el mundo privado para integrar métodos de certificación de barrios sustentables, a pesar de la penetración de certificaciones para edificios. En ese sentido puede que el mercado todavía no visualice las ventajas de la implementación de estos estándares y su posibilidad de generar diferenciación en la demanda y mayores tasas de retorno, como ocurre en el mercado de oficinas. Es posible que al actuar sobre unidades más complejas de análisis y un mercado más diversificado, todavía no emerja la demanda por productos que integren estrategias de sustentabilidad más sofisticadas como sistemas de administración de residuos, eficiencia en el uso de recursos, etc. Podría quizás aventurarse que el mercado está aún consolidando el conocimiento de los beneficios del desarrollo sustentable para productos de oficina y

19

Cámara Chilena de la Construcción, 2014, p. 44. en menor medida de vivienda y es aún temprano para incorporar estas consideraciones a la escala del conjunto o barrio.

Desde el punto de vista público, la prioridad entregada al desarrollo de barrios, ya sean nuevos conjuntos o la rehabilitación de aquellos con obsolescencia física, sigue contando con líneas de financiamiento y apoyos específicos (en el primer caso, los diversos subsidios a la construcción de conjuntos de vivienda social como el Fondo Solidario de Elección de Vivienda D.S. 49, y en el segundo caso los programas Quiero Mi Barrio II y Condominios Sociales de Segunda Oportunidad). Finalmente, desde la comunidad parecieran existir mayores instancias de representación y oportunidades de desarrollo a nivel local que pueden reforzar el crecimiento a partir de barrios sustentables, así como una presión social por mejorar la calidad de vida en ciudades. A todos estos antecedentes habría que sumar la necesidad de integrar políticas y programas que apunten a la generación de ciudades mejor preparadas para responder a catástrofes naturales, a través de la integración de principios de resiliencia contenidos en los estándares revisados, en términos de localización adecuada, estrategias de reducción de riesgo, etc. La mencionada PNDU entrega hoy un marco político que podría sentar la base para el desarrollo de instrumentos más sofisticados de diseño, construcción y evaluación de barrios. 
En consideración de la revisión de antecedentes expuestos en las secciones previas, se postulan una serie de recomendaciones que potencialmente podrían ser usadas en la construcción de un marco de certificación de sustentabilidad para barrios o adaptaciones locales a estándares internacionales. Estas recomendaciones buscan integrar una visión holística y sistema al barrio, para permitir garantizar lineamientos y estrategias de intervención, en particular la promoción del cuidado del medio ambiente y la calidad de vida. Tomando en cuenta lo anterior, se recomienda seguir con esfuerzos posteriores en torno a la definición de 9 áreas temáticas relacionadas con la sustentabilidad de barrios, para desde ahí agruparlas en dimensiones y definir indicadores de medición, con su respectiva priorización de puntaje. Estas áreas temáticas son:

- Recursos hídricos, energéticos y atmosféricos, en términos de sus fuentes, uso y conservación.

- Residuos, buscando establecer sistemas de tratamiento de residuos para aspirar al reciclaje, reutilización y posterior reducción.

- Entorno natural, características del territorio (sitios baldíos, áreas verdes y áreas protegidas).

- Movilidad urbana, en relación a distancia a equipamientos, tiempos de viaje, reducción de dependencia de automóviles y promoción de modos alternativos de transporte.
- Usos, particularmente relacionados con los usos de suelo.

- Aspectos demográficos, nivel de educación de los habitantes, niveles de pobreza y salud de la comunidad.

- Participación ciudadana, relacionada con participación en toma de decisiones y realización de actividades socio-culturales (cultura y ocio).

- Estructura socioeconómica, tasa de desempleo y empleo local.

- Seguridad ciudadana, en términos de percepción de seguridad, infraestructura adecuada e inversión pública.

En definitiva, el desarrollo urbano en Chile se encuentra en una situación expectante: por un lado existen una serie de iniciativas que avanzan hacia un diseño y mantención de edificios "verdes", pero por otro lado existe un área difusa en lo que respecta al entorno de tales proyectos y el espacio usualmente destinado a áreas comunes, calles y parques, que no posee un claro lineamiento en términos de su estándar mínimo u objetivos a aspirar respecto a sus niveles de sustentabilidad. Sin embargo, el de una edificación en su entorno no es un tema menor. A modo de ejemplo, variables simples como un aumento del tráfico vehicular o excesivos conos de sombra han sido difíciles de normar en el 
caso chileno. Esto es aún más preocupante si entendemos que las herramientas actuales de diseño y monitoreo se basan solo en datos extraídos de la condición existente de un proyecto, en un terreno puntual y en un tiempo específico, sin abarcar la enorme complejidad de las dinámicas y fuerzas que pueden detonar en la ciudad.

\section{Bibliografía}

ABDALLA, Gaby; MAAS, Ger; HUYGHE, Jules y 00STRA, Mieke. Criticism on environmental assessment tools. International Proceedings of Chemical, Biological and Environmental Engineering. 6: 443-446, 2011. ISSN 2010-4618.

BRAUNGART, Michael y MCDONOUGH, William. Cradle to cradle. Madrid, McGraw Hill. 2005. 208 p. ISBN 9788448142957.

BREEAM ES Urbanismo. [En línea]. BREEAM. 2013. [Fecha de consulta: 15 febrero 2015]. Disponible en: http://www.breeam.es/index.php/extranetbreeam/extranet/manuales-tecnicos-breeam/ urbanismo/detail.

CÁMARA Chilena de la Construcción. Mercado inmobiliario nacional. [En línea]. Informe MACH Macroeconomía y Construcción. (41): 43-45, diciembre 2014. [Fecha de consulta: 15 febrero 2015]. Disponible en: http://www.cchc.cl/centro-deinformacion/publicaciones/publicaciones-mach/ informe-mach-41-1.
DÍAZ, Luis. Criterios operativos para el desarrollo sustentable. En DALY, H. y SCHUTZE, C. Crisis ecológica y sociedad. Valencia, España. 2011.

GARDE, Ajay. Sustainable by design?: insights from U.S. LEED-ND pilot projects. Journal of the American Planning Association. 75, p. 424-440. 2009.

GREEN Star Communities. [En línea]. Green Building Council of Australia. 2013. [Fecha de consulta: 13 junio 2014]. Disponible en: http://www.gbca.org. au/green-star/green-star-communities/.

HAAPI0, Appu. Towards sustainable urban communities. Environmental Impact Assessment Review. 32(1): [165-169], 2012. DOI 10.1016/j.eiar.2011.08.002.

KELLETT, Ronald. Sustainability indicators for computer-based tools in community design, 1. Ottawa, Canada Mortgage and Housing Corporation. 2009.

LAM, Patrick T.I.; CHAN, Edwin; POON, C.S.; CHAUC, C.K. y CHUN, K.P. Factors affecting the implementation of green specifications in construction. [En línea]. Journal of environmental management. 91(3): 654-61, 2010. [Fecha de consulta: 13 junio 2014]. DOI 10.1016/j.jenvman.2009.09.029.

MÁRQUEZ, Francisca. El retorno del barrio. [En línea]. Mensaje. 55(555), 38-41, 2006. [Fecha de consulta: 10 marzo 2015]. Disponible en: http://biblioteca.uahurtado.cl/UJAH/msj/docs/2006/n555_38. pdf.

ONE Planet Living. [En línea]. Bioregional. S.f. Disponible en: http://www.bioregional.com/oneplanetliving/. 
RUEDA, Salvador. Ecología urbana. Barcelona, Beta. 1995. 266 p. ISBN 8470913573.

SCHWEBER, Libby. The effect of BREEAM on clients and construction professionals. Building Research and Information. 41(2): 1-17, 2013. ISSN 14664321. DOI 10.1080/09613218.2013.768495

SHARIFI, Ayyoob y MURAYAMA, Akito. A critical review of seven selected neighborhood sustainability assessment tools. Environmental Impact Assessment Review. 38: 73-87, 2013. DOI 10.1016/j. eiar.2012.06.006.

U.S. Green Building Council. LEED 2009 for Neighborhood Development. [En línea]. U.S. Green Building Council. 2014. [Fecha de consulta: 11 febrero 2014]. Disponible en: http://www.usgbc.org/resources/leed-neighborhood-development-v2009current-version.

ZU0, Jian y ZHA0, Zhen-Yu. Green building researchcurrent status and future agenda: A review. [En línea]. Renewable and Sustainable Energy Reviews. 30: 271-281, 2014. ISSN 1364-0321. [Fecha de consulta: 11 diciembre 2014]. DOI 10.1016/j. rser.2013.10.021. 